\title{
Benefícios da Moringa oleifera para saúde humana e animal: Revisão de Literatura
}

\author{
Benefits of Moringa oleifera for human and animal health: Literature review \\ Beneficios del aceite de Moringa oleifera para la salud humana y animal: Revision de literatura
}

Recebido: 23/06/2021 | Revisado: 01/07/2021 | Aceito: 06/07/2021 | Publicado: 16/07/2021

\author{
Maria Vitória Serafim da Silva \\ ORCID: https://orcid.org/0000-0001-5616-5662 \\ Universidade Federal do Rio Grande do Norte, Brasil \\ E-mail:mariavitoriazoo@hotmail.com \\ Rodrigo Tenório Padilha \\ ORCID: https://orcid.org/0000-0001-8737-976X \\ Rodrigo Tenório Padilha Limitada, Brasil \\ E-mail: rodrigopadilhavet@yahoo.com.br \\ Deborah de Melo Magalhães Padilha \\ ORCID: https://orcid.org/0000-0002-6428-8627 \\ Universidade Potiguar, Brasil \\ E-mail: dmmvet@hotmail.com
}

\begin{abstract}
Resumo
Moringa oleifera Lam. é uma espécie de planta pertencente à família Moringaceae, arbórea, originada do Nordeste indiano. Por apresentar várias aplicações, desde a saúde humana e animal até a utilização para fins ambientais, como a purificação de águas, essa espécie é cultivada no mundo inteiro. Das folhas às raízes, essa planta apresenta um alto valor medicinal. Vários estudos comprovaram os efeitos terapêuticos da Moringa oleifera Lam., dentre eles: potente anti-inflamatório, redução da glicose, anticolesterol, hepatoprotetor, anticâncer e propriedades antimicrobianas. Tendo em vista a importância da Moringa oleifera Lam. como um fitoterápico, o objetivo deste artigo de revisão é apresentar os diversos benefícios desta planta na saúde humana e animal. Portanto, neste artigo foi realizada uma revisão integrativa, sendo estudados um total de 63 artigos científicos, nos idiomas inglês, espanhol e português. Com base nos estudos avaliados, foi confirmado que, várias partes da Moringa oleifera Lam. apresentam efeitos terapêuticos, como as folhas, sementes, flor e raízes. Esses efeitos foram atribuídos as substâncias fitoconstituintes que elas apresentam, como flavonóides, fenóis, alcalóides, taninos, saponinas, glucosinalatos, fitatos, oxalatos, proteínas e vitaminas. Sua ação terapêutica, e sua eficácia na resposta aos tratamentos, dependem das formas de apresentação e da dose administrada.
\end{abstract}

Palavras-chave: Funções terapêuticas; Fitoterápico; Moringa; Nutracêutico.

\begin{abstract}
Moringa oleifera Lam. It is a species belonging to the Moringaceae family, arboreal, cultivated all over the world, for presenting several applications, from human and animal health to use for environmental purposes, such as water purification. From the leaves, the roots of the plant have a high medicinal value and several studies have proven their therapeutic effects, such as powerful anti-inflammatory, glucose reduction, anti-cholesterol, hepatoprotective, anticancer and antimicrobial properties. The purpose of this review article is to present the various benefits of Moringa oleifera in human and animal health. Therefore, an integrative review was carried out in this article, and a total of 63 scientific articles were studied, in English, Spanish and Portuguese. Based on the studies reviewed, it was confirmed that several parts of the plant have therapeutic effects, such as leaves, seeds, flowers and roots have phytochemicals, such as flavonoids, phenols, alkaloids, tannins, saponins, glucosinalates, phytates, oxalates, proteins and vitamins, responsible for their therapeutic action, and their effectiveness in responding to treatments, depends on the forms of presentation and the dose administered.
\end{abstract}

Keywords: Therapeutic functions; Phytotherapic; Moringa; Nutraceutical.

\section{Resumen}

Moringa oleifera Lam. Es una especie perteneciente a la familia Moringaceae, arbórea, cultivada en todo el mundo, por presentar diversas aplicaciones, desde la salud humana y animal hasta su uso con fines ambientales, como la depuración de aguas. A partir de las hojas, las raíces de la planta tienen un alto valor medicinal y varios estudios han demostrado sus efectos terapéuticos, como potentes propiedades antiinflamatorias, reductoras de glucosa, anticolesterol, hepatoprotectoras, anti-cancerígenas y antimicrobianas. El propósito de este artículo de revisión es presentar los diversos beneficios de Moringa oleifera en la salud humana y animal. Por ello, en este artículo se realizó una revisión integradora y se estudiaron un total de 62 artículos científicos, en inglés, español y portugués. Con base en los estudios revisados, se confirmó que várias partes de la planta tienen efectos terapéuticos, como hojas, semillas, flores y raíces tienen fitoquímicos, como flavonoides, fenoles, alcaloides, taninos, saponinas, glucosinalatos, fitatos, 
oxalatos, proteínas y Las vitaminas, responsables de su acción terapéutica y su eficacia en la respuesta a los tratamientos, depende de las formas de presentación y de la dosis administrada.

Palabras clave: Funciones terapéuticas; Fitoterápico; Moringa; Nutracéutico.

\section{Introdução}

A Moringa oleifera possui grande interesse econômico, medicinal e industrial (Mbikay, 2012). É popularmente conhecida como árvore de coxa ou raiz-forte, originária do norte da Índia e da África. Devido as suas propriedades nutricionais é também conhecida como 'Árvore Milagrosa'. Em todas as partes da árvore moringa, incluindo frutas, sementes, folhas, flores, casca e raízes, foram encontradas grandes quantidades de nutrientes benéficos (Dhakad et al., 2019; Zaku et al., 2015).

De acordo com Ademiluy et al. (2018) e Gopalakrishnan et al. (2016), a folha de moringa é uma fonte eficaz de antioxidantes naturais, incluindo ácidos fenólicos, flavonóides, vitamina C, tanino, saponina, fitato, oxalato, alcalóides, cardenolidos e glicosídeos cardíacos. Assim, essa planta não só fornece nutrientes essenciais à nutrição, como também possui vários efeitos terapêuticos medicamentosos, incluindo anti-fibrótico, anti-inflamatórios, anti-microbianos, antihiperglicémicos, anti-oxidantes, anti-tumoral, anti-cancro e anti-clastogênico (Mohanty et al., 2021; Abdull et al., 2014).

A moringa tem sido usada desde o ano150 d.C., por antigos reis e rainhas em sua dieta para manter mente e pele saudáveis (Fahey et al., 2005). As folhas, vagens, sementes, gomas, casca e flores de Moringa são usadas em mais de 80 países para aliviar deficiências minerais e vitamínicas, fortalecer o sistema cardiovascular, promover níveis normais de glicose no sangue, neutralizar e reduzir a malignidade dos radicais livres (Mohanty et al., 2021; Mahmood et al., 2010).

Diante das importantes propriedades da Moringa para medicina humana e animal, o presente artigo objetivou demonstrar os principais efeitos dessa planta como antiinflamatório, antiglicêmica, anticolesterol e anticâncer, ressaltando os benefícios da Moringa oleifera na saúde humana e animal.

\section{Metodologia}

A presente revisão de literatura é baseada no estado da arte, uma revisão integrativa qualitativa (Proetti et al., 2017), tendo como bases de dados científicos PubMed, Scielo e Google Acadêmico. As palavras-chave utilizadas para coleta de informações bibliográficas foram: "Moringa oleifera"; "Moringa oleifera -fitoterápico", "Moringa oleifera e funções terapêuticas". Este tipo de revisão é um método minucioso que produz os resultados encontrados na pesquisa de forma sistemática.

Para este artigo foram empregados um total de 62 artigos científicos, datados, em sua maioria, de 2005 a 2021 . Para construção dessa revisão, os critérios de inclusão foram artigos científicos, resenhas de artigos científicos e ensaios clínicos em línguas portuguesa, inglesa e espanhola. Além disso, teve como critérios de exclusão capítulos de livros, editoriais, e outros formatos de textos que não passaram por processo rigoroso de avaliação.

\section{Resultados e Discussão}

\subsection{Moringa Oleifera}

Espécie perene, da família Moringaceae, a Moringa oleifera, conhecida popularmente como Moringa, é originária do Himalaia, amplamente distribuída na Índia, Egito, Filipinas, Ceilão, Tailândia, Malásia, Paquistão, Singapura, Jamaica e Nigéria, cultivada em áreas tropicais e subtropicais (Figura 1), tratando-se de uma espécie pan-tropical (Aguiar, 2010; Macedo et al., 2010). Esta planta se adapta tanto às condições irrigadas quanto às de sequeiro, pouco exigente em solos e fertilizantes. No Brasil, a moringa encontra-se disseminada na região Nordeste, mas tem sua expansão em todo o território brasileiro. 
A Moringa oleifera Lam. é caracterizada como uma árvore pequena ou média, com cerca de $10 \mathrm{~m}$ de altura, de multiuso, usada como especiaria, fonte de óleo de cozinha e cosmético, além de ser utilizada como planta medicinal (Mohanty et al., 2021). Tem contribuído na prevenção de doenças e desnutrição, principalmente por ser de fácil cultivo e baixo custo. As sementes são de cor marrom escura, aladas, possuindo, cada uma, 3 asas (Schuarz, 2000; Foild et al., 2003), ricas em proteínas $(33,90 \%)$ e lipídeos $(37,20 \%)$ (Muyibi et al., 2002). O óleo extraído desta planta contém elevados teores de ácidos graxos insaturados, em especial o oleico (71,6\%), o palmítico e o behênico (Lalas \& Tsakins, 2002). A raiz apresenta-se em forma de tubérculo e tem a função de armazenar energia para a planta (Macedo et al., 2010). Na aparência e no sabor, a moringa assemelha-se ao rabanete. A casca da raiz é espessa, mole e reticulada, apresentando cor pardo clara, externamente, e branca, internamente (Cysne, 2006).

Figura 1. Árvore, folhas, flor, fruto e sementes da Moringa oleífera. A foto localizada mais à direita ilustra o pó extraído das folhas da moringa.

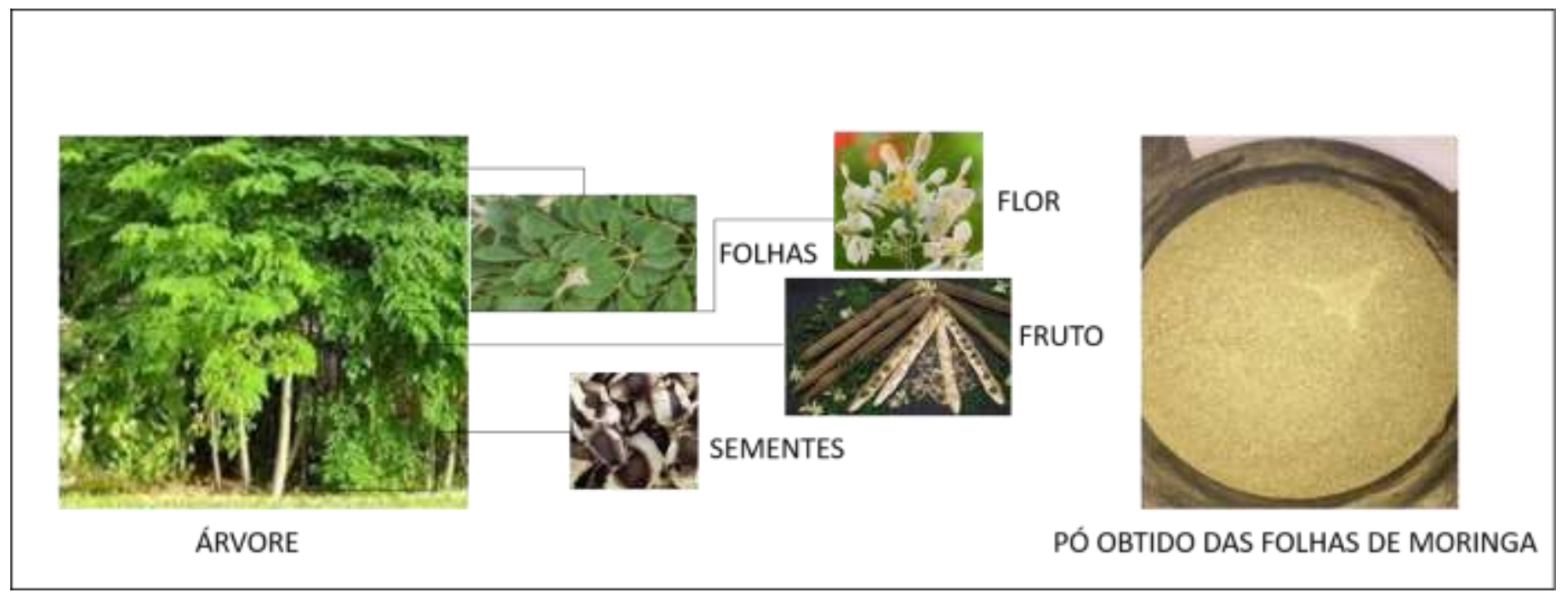

Fonte: Autores.

Na Figura 1 é importante destacar a dimensão da árvore da Moringa oleífera e a característica de cada parte da planta. O pó obtido das folhas de moringa ilustrado na figura, geralmente é a substância que se utiliza para fazer os fitoterápicos para ser consumido pelos seres humanos e animais.

\subsection{Aplicação terapêutica}

A Moringa oleifera tem seu efeito terapêutico comprovado em diversos estudos atualmente revisados (Mohanty et al., 2021). Os flavonóides presentes nas folhas dessa planta possuem ação antiinflamatória, hepatoprotetora, antioxidante, antidiabético e neuroprotetor (Leone et al., 2016). Nas folhas e sementes da Moringa, os glicosídeos de tiocarbamatos e isotiocianatos têm um efeito hipotensor e imunológico. Além disso, em sua raiz, a presença da perigospermina tem um efeito antibacteriano e antifúngico.

Recentemente, a Moringa oleifera demonstrou sua ação anti-inflamatória em células epiteliais mamárias em bovinos (Cheng et al., 2019). Além disso, em outro estudo, a Moringa demonstrou efeitos antidiabéticos e antioxidantes contra os danos prejudiciais do estresse oxidativo e complicações diabéticas (Gutiérrez et al., 2018) e, ainda, melhorou o desempenho reprodutivo em camundongos (Zeng et al., 2019). 


\subsubsection{Atividade antimicrobiana}

A Moringa apresenta diversas moléculas com atividades antimicrobianas. Dentre essas moléculas, destaca-se o Benzil-isotiocianato (BITC), pertencente ao grupo dos isotiocianatos. Estes compostos são originados a partir da hidrólise dos glucosinolatos, também conhecidos como glicosídeos do óleo de mostarda, e são liberados na planta após alguma lesão tecidual (Eilert, Wolter, \& Nahrstedt, 1981; Matu \& Van Staden, 2003).

A pterigospermina é outra molécula com atividade antimicrobiana presente nas raízes da moringa. Foi relatado, em estudos preliminares realizados na década de 1950 (Kurup \& Rao, 1954a) que a pterigospermina é resultado da condensação de duas moléculas de benzil-isotiocianato e uma de benzoquinona, e que essa substancia podia interferir diretamente no metabolismo bacteriano do ácido glutâmico (Kurup \& Rao, 1954b). Outras substâncias presentes nos tecidos da Moringa também são conhecidas por inibir o crescimento microbiano, como os glicosídeos saponinas, niazirina, niaziridina, polifenóis (flavonoides) e alcaloides (moringina e moringinina) (Jahn, Musnad \& Burgastaller, 1986; Doughari \& Pukuma, 2007; Shanker, Gupta \& Srivastava, 2007).

Os pigmentos flavonoides kaempferol, ramnetina, isoquercetina e kaempferitrina foram encontrados na moringa (Anwar et al., 2007). Os flavonoides são compostos polifenólicos que têm por base a estrutura da flavona (2-fenilbenzopirona) e possuem efeitos bacteriostáticos ou bactericidas (Silva, 2010; Rego, 2012). Além dessas propriedades, também podem inibir a formação de biofilmes bacterianos (Miller \& Bassler, 2001).

Um estudo detectou a presença de dois alcaloides, denominados moringina e moringinina na casca do caule da moringa (Fatima et al., 2014). Os alcaloides estão presentes na natureza como compostos nitrogenados em plantas, fungos e bactérias e já deram origem a fármacos antimicrobianos, como o metronidazol e quinolonas (Qiu et al., 2014; Cushnie et al., 2014).

A moringa também apresentou uso potencial contra espécies de patógenos tolerantes aos antibióticos convencionais, demonstrando atividade contra cepas multirresistentes (Arora \& Onsare, 2014).

As folhas, raízes, cascas e sementes de Moringa oleifera apresentaram atividade antimicrobiana contra bactérias e fungos. A planta mostrou atividade in vitro contra bactérias, leveduras, dermófitos e helmintos através do método de difusão em disco, as folhas frescas e o extrato aquoso de sementes inibiram o crescimento de Pseudomona aeruginosa $e$ Staphylococcus aureus (Cáceres, 1991). Além disso, o extrato da moringa também exibiu atividade antibacteriana em bactérias associadas à diarreia: Serratia marcescens, shigella dysenteriae, Enterobacter sp., E.coli, Klebssiella pneumoniae e Salmonella sp, (Rahman, 2010).

Em 2011, Rocha e colaboradores, trabalhando com extratos de Moringa oleifera e Vernonia sp. sobre Candida albicans e Microsporum canis isolados de cães e gatos observaram atividade antifúngica ante as cepas de C. albicans e M. canis in vitro e baixa toxicidade.

Devido à resistência microbiana ser um desafio para a medicina humana e Veterinária, a Moringa mostrou-se promissora, tendo em vista que os fitoquímicos presentes demonstram forte atividade antimicrobiana contra diversas espécies de microrganismos. Além disso, esses compostos também podem ser utilizados como agentes modificadores de resistência, aumentando a eficácia dos antibióticos ao inibir os mecanismos de resistência dos microrganismos patogênicos (Rocha et al., 2011).

\subsubsection{Atividade anti-inflamatória}

A Moringa apresenta 36 compostos que foram isolados e estão relacionados à atividade anti-inflamatória, entre os quais destacam-se: alcalóides, glucosinolatos, isotiocianatos, flavonóides, ácidos fenólicos, esteróis e vitaminas (Martín et al., 2013). Estudos demonstraram que a quercetina está envolvida na redução do processo inflamatório por inibir a ativação do 
fator nuclear kappa-beta (NF-к $\beta$ ), que é uma etapa essencial para desencadear o processo inflamatório (Brilhante et al., 2017; Vergara-Jimenez et al., 2017).

O efeito antiinflamatório com extrato de folhas de Moringa foi comprovado em um modelo de edema plantar induzido por carragena em ratos Sprague-Dawley. Os animais receberam tratamento por via intraperitoneal do extrato nas doses de 10, $30,100 \mathrm{mg} / \mathrm{Kg}, 30$ minutos antes de administrar carragenina. O resultado confirmou que o extrato de Moringa oleifera mostrou atividade antiinflamatória em todas as doses aplicadas, embora tenha sido menos eficaz em comparação ao ibuprofeno, em uma dose de tratamento de $100 \mathrm{mg} / \mathrm{Kg}$ (Sulaiman, 2008).

\subsubsection{Anticâncer}

Uma outra ação da Moringa oleifera é na atividade anticâncer. Poderosa atividade antitumoral foi identificada nos extratos etanólicos de folhas e sementes de Moringa oleifera, comprovados por Faizi (1994), sendo identificados tiocarbamato e isotiocianato, que atuam como inibidores do promotor do tumor.

Estudos recentes demonstram o potencial da $\mathrm{MO}$ no tratamento anticâncer, na qual a administração de extrato aquoso de MO reduziu o tamanho de tumor em camundongos e aumentou o tempo de vida dos animais na qual ela foi administrada (Bahoi et al., 2020).

\subsubsection{Hepatoprotetor}

O efeito hepatoprotetor da Moringa oleifera tem sido relacionado a sua composição de flavonóides (quercetina, kaempferol, mirecetina) e compostos fenólicos (catequina, epicatequina, ácido ferúlico, ácido elágico) (Mallya, 2017). Extratos das folhas também demonstraram prevenir a toxicidade do fígado devido ao paracetamol. Neste mesmo experimento, foi observado que a administração de 200 e $800 \mathrm{mg} / \mathrm{Kg}$ de extratos aquosos de etanol de folhas de Moringa oleifera preveniram danos ao fígado induzido por acetaminofeno, diminuiu as aminotransferases e aumentou a glutationa do fígado.

\subsubsection{Antidiabética}

Um corpo crescente de evidências experimentais e epidemiológicas sugere que a Moringa oleifera Lam tem efeitos antidiabéticos e antioxidantes contra os danos prejudiciais do estresse oxidativo e complicações diabéticas (Abd Eldaim et al., 2016; Vergara et al., 2017). As atividades benéficas de Moringa no metabolismo de carboidratos têm sido demonstradas por diferentes processos fisiológicos, incluindo a prevenção e restauração da integridade e função das células $\beta$ pancreáticas, aumentando a ação da insulina, melhorando a captação e utilização de glicose, dentre outros (Makkar et al., 1996).

Um estudo observou que, após a administração de $200 \mathrm{mg} / \mathrm{Kg}$ de extrato aquoso de Moringa oleifera por 60 dias, houve uma diminuição na concentração de glicose no sangue do grupo de ratos diabéticos induzidos por glicose resistentes à insulina (IR) e resistentes a estreptozotocina (STZ) (Divi, 2012).

As folhas dessa planta também regularam os níveis de glicose em estudos realizados em ratos, no qual aqueles que receberam glicose com farinha da folha de Moringa oleifera mantiveram os níveis mais baixos de glicose em comparação com aqueles que ingeriram apenas glicose. A atividade reguladora da glicose pode ser devido ao alto teor de substâncias polifenólicas (quercetina e kempferol) (Olson, 2013).

\subsubsection{Cardiovascular e hipolipemiante}

O extrato etanólico das folhas de Moringa oleifera apresentou uma importante atividade anti-hipertensiva ou hipotensora. Um experimento in vitro foi realizado no coração do animal e glicosídeos de tiocarbamatos e isotiocianatos foram identificados responsável pela potente atividade hipotensora (Gilani, 1994). 
Para indivíduos com arteriosclerose, a ingestão de 2g de folhas de Moringa oleifera fresca diariamente mostrou efeitos positivos no tratamento. (Valere, 2015).

Estudos demonstraram que compostos fenólicos de Moringa diminuiu significativamente o colesterol intracelular total, inibiu a atividade da HMG CoA redutase (3-hidroxi-3-metil-glutaril-coenzima A redutase) e aumentou a atividade de ligação ao receptor da Lipoproteína de Baixa Densidade (LDL) em células HepG2 (Tabbom et al., 2016). Além disso, foi relatado que a Moringa durante a adipogênese melhorou a funcionalidade dos adipócitos e aumentou a expressão da proteína desacopladora 1 (UCP1), sirtuína 1 (SIRT-1) e do coativador 1-alfa do receptor ativado por proliferador de peroxissoma (PGC$1 \alpha)$ envolvido na termogênese, modulando o metabolismo lipídico (Borbagalho et al., 2015).

\subsubsection{Perspectivas futuras para utilização da Moringa oleífera}

Nos últimos anos, tem-se observado o uso crescente de alimentação natural e nutracêuticos em medicina veterinária, para auxiliar no tratamento clínico (Zaine et al., 2014). Além da dieta atender as exigências nutricionais do animal, estuda-se a possibilidade desta alimentação promover a prevenção de doenças ou, até mesmo, auxiliar no tratamento das mesmas pelo uso de nutracêuticos, compostos que podem ser definidos como: "um alimento (ou parte de um alimento) que fornece benefícios médicos ou à saúde, incluindo a prevenção e/ou tratamento de uma doença" (Brower, 1998) ou "uma substância produzida em uma forma purificada que, quando administrada oralmente a pacientes, tem como objetivo prover a eles os elementos para sua estrutura e função normal, para melhorar a saúde e o bem-estar” (Boothe, 1997).

Atualmente, se reconhece a importância do manejo alimentar com formulação de dieta específica para algumas doenças que induzem alterações metabólicas e funcionais específicas. A dieta é formulada de modo a apresentar composição nutricional que melhor se adeque às modificações metabólicas induzidas pela doença (Hand et al., 2010).

Acredita-se que o uso de nutracêuticos, em associação a estas dietas específicas, possa resultar em benefícios adicionais aos pacientes. No entanto, para a maioria das substâncias, ainda existe pouca informação científica e muita controvérsia sobre sua eficácia e dosagem adequadas. Estas informações são especialmente escassas para Pets, principalmente cães e gatos, espécies na qual pouca pesquisa foi ainda produzida.

Neste contexto, a Moringa oleifera surge como um futuro promissor pelos nutrientes que possui, principalmente no que diz respeito ao seu poder antiinflamatório e antimicrobiano, sendo uma alternativa para ser avaliada em cães que apresentam a dermatite atópica canina.

De acordo com Kersey e colaboradores (2013), a Dermatite atópica (DA) é uma das doenças que mais acometem os cães (Figura 2). É definida como uma doença dermatológica que apresenta prurido e inflamação através da produção de imunoglobulina E (IgE) que atinge predominantemente antígenos ambientais (Halliwell, 2006). A DA pode afetar severamente a qualidade de vida de um animal de estimação. Sendo o segundo distúrbio cutâneo alérgico mais comum, a DA é menos frequente apenas que a dermatite alérgica à picada de pulgas (White, 1998; Hillier \& Griffin, 2001; Sousa \& Halliwell, 2001; Scott, Miller \& Griffin, 2001). 
Figura 2. Cão apresentando dermatite atópica na região da flexura anticubital.

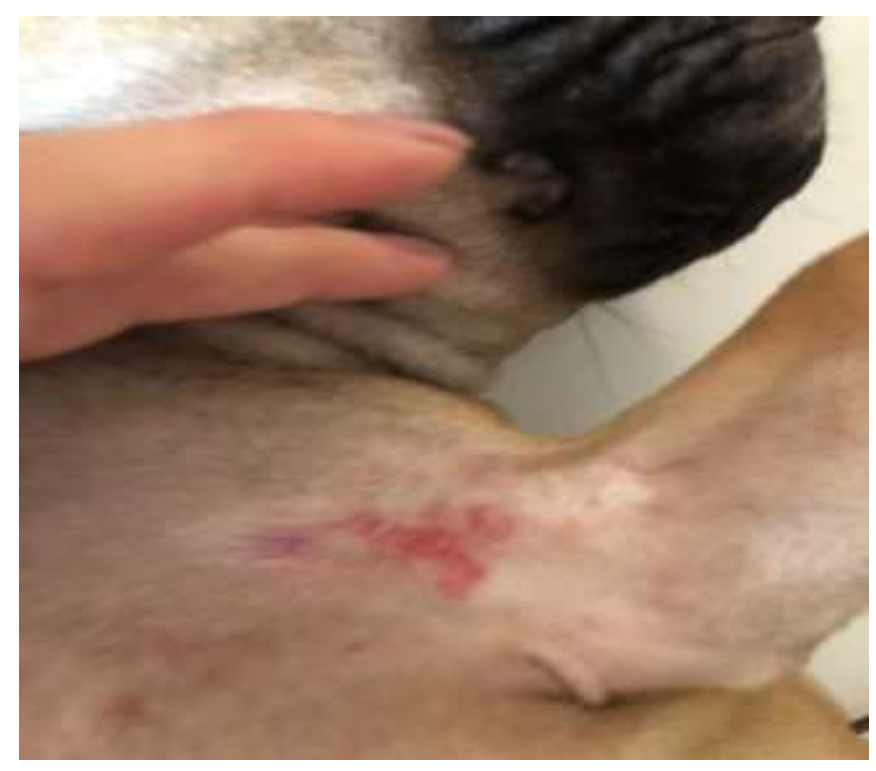

Fonte: Autores.

Na Figura 2 é importante observar na DA uma lesão com aspecto eritematoso, papular e com colarete epidérmico na região da flexura anticubital. Além disso, também é possível observar uma alopecia na região afetada pela DA.

O tratamento da DA é multifatorial e, tipicamente, requer modulação imune combinada com a evitação de alérgenos e fortalecimento da barreira epidérmica (Olivry et al., 2010). Embora o papel da nutrição na DA canina precise de mais elucidação, vários estudos demonstram que a suplementação oral com ácidos graxos poliinsaturados (PUFA) melhora o prurido e pode reduzir as dosagens de glicocorticoides e ciclosporina necessárias para controlar os sinais clínicos (Logas et al., 1994; Muller et al., 2016).

Antibióticos tópicos e orais são frequentemente usados em infecções bacterianas caninas de pele, ouvidos e feridas, e a ocorrência de resistência é uma grande preocupação. Um aumento da resistência antimicrobiana em bactérias do grupo Staphylococcus intermedius, isoladas de amostras clínicas de cães e gatos, tem sido relatado (Beever et al., 2015). Achados semelhantes foram relatadas para P. aeruginosa (Arais et al., 2016).

$\mathrm{O}$ tratamento de infecções associadas a bactérias resistentes aos antimicrobianos pode ser muito desafiador. Por essa razão, o desenvolvimento e a avaliação dos efeitos de um petisco fitoterápico a base de folhas de Moringa oleifera sobre a DA seria de grande importância para auxiliar na prevenção e no tratamento dessa doença, reduzindo desta forma o uso de antiinflamatórios, corticóides e antibióticos, diminuindo assim o surgimento de resistência microbiana e permitindo uma vida mais longeva a esses animais.

\section{Considerações Finais}

O presente artigo relata a importância da Moringa oleifera para os diversos fins terapêuticos na medicina humana e animal, por apresentar efeitos anti-inflamatórios, redução de glicose, anticolesterol, hepatoprotetor, anticâncer e propriedades antimicrobianas.

Os diversos componentes da Moringa encontrados nos artigos atualmente revisados, comprovam seu potencial terapêutico, como por exemplo: os flavonóides presentes nas folhas da planta que possui propriedade antiinflamatória, hepatoprotetor, antioxidante, antidiabético e neuroprotetor. Nas folhas e sementes da planta, a presença dos glicosídeos de 
tiocarbamatos e isotiocianatos com seu efeito hipotensor e imunológico e a perigospermina presente na raiz da planta tem um efeito antibacteriano e antifúngico.

Dessa forma, a Moringa oleifera mostra-se como uma planta oportuna para aplicação em pesquisas com outras doenças, além de ser uma opção inovadora no desenvolvimento de pesquisas com pets, voltada para sua avaliação no tratamento de umas das principais doenças que acometem a qualidade de vida e a longevidade destes, a dermatite atópica canina.

Diante de todas as propriedades da Moringa oleifera, principalmente as ações antiinflatatória, antioxidante e antimicrobiana, trabalhos futuros com a utilização desta planta no consumo de animais que possuam dermatite atópica poderiam ser de grande valia para prevenção da DA ou até mesmo a diminuição dos sintomas causados por essa patologia.

\section{Agradecimentos}

Os autores agradecem ao órgão de fomento Conselho Nacional em Pesquisa (CNPq) pelo apoio as pesquisas realizadas pelo grupo e pela bolsa de produtividade da professora Deborah de Melo Magalhães Padilha.

\section{Referências}

Abd, M. A., Shaban, A. E. A., \& Abd, E. S. A. (2017). An aqueous extract from Moringa oleifera leaves ameliorates hepatotoxicity in alloxan-induced diabetic rats. Biochem. Cell Biology, 95, 524-530.

Ademiluyi, A, O., et al. (2018). Drying alters the phenolic constituents, antioxidante properties, amylase, and glucosidase inhibitory properties of Moringa (Moringa oleifera) leaf. Food Science Nutrition, 6, 2123-2133.

Abdull, R., Ahmad, F., Ibrahim, M. D., \& Kntayya, S. B (2014). Health benefits of Moringa oleifera. Asian Pacific Journal of Cancer Prevention, 15, 85718576.

Aguiar, F. L. N. (2010) Avaliação do potencial antifúngico de produtos de plantas em cepas de Candida albicans e Microsporum canis isolados de cães e gatos: um destaque para Moringa oleifera e Vernonia sp. 2010. 63f. Dissertação (Mestrado em Ciências Veterinárias) - Universidade Estadual do Ceará Fortaleza.

Anwar, F., et al. (2007). Moringa oleifera: a food plant with multiple medicinal uses. Phytother Reserach, 21(1),17-25.

Arais, L. R., et al. (2016). Antimicrobial resistance, integron carriage, and gyrA and gyrB mutations in Pseudomonas aeruginosa isolated from dogs with otitis externa and pyoderma in Brazil. Veterinary Dermatology, 27(2), 113-117.

Arora, D. S., \& Onsare, J. G (2014). In vitro antimicrobial evaluation and phytoconstituents of Moringa oleifera pod husks. Industrial Crops and Products, 52, $125-135$.

Barhoi, D ., et al. (2020). Aqueous Extract Of Moringa Oleifera Exhibit Potential Anticancer Activity And Can Be Used As A Possible Cancer Therapeutic Agent: A Study Involving In Vitro And In Vivo Approach. Journal Of The American College Of Nutrition, 40, 70-85.

Barbagallo, I., Venella, L., Cambria, M. T., Tibullo, D., Godos, J., Guarnaccia, L., Zapalla, A., Galvano, F \& Li Volti, G (2015). Silibinin regulates lipid metabolism and differentiation in functional human adipocytes. Frontiers in Pharmacology, 6, 309.

Beever, L., Bond, R., Graham, P. A., Jackson, B., Lloyd, D. H., \& Loeffler, A (2015). Increasing antimicrobial resistance in clinical isolates of Staphylococcus intermedius group bacteria and emergence of MRSP in the UK. Veterinary Record, 176(7), 172.

Brilhante, R. S. N., et al. 2017). Research advances on the multiple uses of Moringa oleifera: A sustainable alternative for socially neglected population. Asian Pacific Journal of Tropical Medicine, 10(7), 621-630.

Boothe, D. M. (1997). Nutraceuticals in veterinary medicine, part 1: definitions and regulations. Compendium on Continuing Education for the Practicing Veterinarian, 19(11), 1248-1255.

Brower, V. (1998). Nutraceuticals: poised for a healthy slice of the healthcare market? Nature Biotechnology, 16(8), 728-731.

Cáceres, A. O., et al. (1991). Propriedades farmacológicas de Moringa oleifera: detección preliminar de actividad antimicrobiana. $33(3), 213$.

Cheng, W. N., et al. (2019). Moringa extract attenuates inflammatory responses and increases gene expression of casein in bovine mammary epithelial cells. Revista Animals, 9(7), 391.

Cysne, J. R B. (2006). Propagação in vitro da Moringa oleifera Lam. 2006. 81 f. Dissertação (Mestrado). Universidade Federal do Ceará - UFC, Ceará.

Cushnie, T. P. T., et al. (2014). Alkaloids: and overview of their bacterial, antibiotic-enhancing and antivirulence activities. International Jounal of Antimicrobial Agents, 44 (5), 377-386. 
Divi, S. B. D. S. (2012). Evaluación del potencial antidiabético y antihiperlipedémico zdel extracto acuoso de Moringa oleifera en ratas diabéticas resistentes a la insulina alimentadas con fructosa e inducida por STZ: un estudio comparativo. Asian Journal of Pharmaceutical and Clinical Research, 5, 67-72.

Doughari, J. H., et al. (2007). Antibacterial effects of Balanites aegyptiaca L. Drel. and Moringa oleifera Lam. on Salmonella thyphi. African J Biotechnology, $6(19), 2212-2215$.

Ercole, F. F., et al. (2014). Revisão integrativa versus revisão sistemática. Revista Mineira de Enfermagem, 18(1), 9-12.

Eilert, U., Wolter, B., \& Nahrstedt, A (1981). The antibiotic principle of seeds of Moringa oleifera and Moringa stenopetala. Planta Medicinal, 42(1), 55-61.

Fahey, J (2005). Moringa oleifera: A review of the medical evidence for its nutritional, therapeutic, and prophylactic properties. Part 1. Trees for life Journal, $157-164$.

Faizi, S. S. B. R. (1994). Elucidación del aislamiento y la estructura de nuevos glucósidos de nitrilo y aceite de mostaza de Moringa oleifera y su efecto sobre la presión arterial. Jounal of Natural Products, 57, 1256-1261.

Fatima, T., et al. (2014). Phytomedicinal value of Moringa oleifera with special reference to antiparasitics. Pak. J. Agri Sciences, 51(1), 251-262.

Foild, N., Mayorga, L., \& Vásquez, W. (2003). Utilización del marango (Moringa oleifera) como forraje fresco para ganado. Universidad Nacional de Ingeniería, Manágua, Nicarágua.

Gilani, A. H., \& Aksasssrs, B. (1994). Estudios farmacológicos hipotensivas y espasmolíticas sobre actividades de compuestos puros de Moringa oleifera. Phytotherapy Research, 8(2), 87-91.

Gopalakrishnan, L., Doriya, K., \& Kumar, D. S (2016). Moringa oleifera: A review on nutritive importance and its medicinais application. Food Science and Human Wellness, 5, 49-56.

Halliwell, R. (2006) Revised nomenclature for veterinary allergy. Veterinary Immunology and Immunopathology, 114(3), 207-8.

Hand, M. S., et al. (2010). Small animal clinical nutrition. 5. ed. Topeka, KS: Mark Morris Institute, 1314.

Hillier, A., \& Griffin, C. E (2001). The ACVD task force on canine atopic dermatitis (I): incidence and prevalence. Veterinary Immunology and Immunopathology, 81(3-4), 147-151.

Jahn, S. A., Musnad, H., \& Burgastaller, H (1986). Tree that purifies water: Cultivating multipurpose Moringaceae in the Sudan. Unasylva, 38(152), 23-28.

Kersey, K. M., et al. (2013). Emergências dermatológicas: identificação e tratamento. Compend (Yardley, PA), 35.

Kurup, P. A., \& Rao, P. L. N. (1954a.) Antibiotic principle from Moringa pterigosperma. Part II. Chemical nature of pterygospermin. Indian Journal of Medical Research, 42(1), 85-95.

Kurup, P. A., \& Rao, P. L. N. (1954b). Antibiotic principle from Moringa pterigosperma. Part V. effect of pterygospermin in the assimilation of glutamic acid by micrococcus pyogenes var. aureus. Indian Journal of Medical Research, 42(1), 109-114.

Lalas, S., \& Tsaknis, T (2002). Caracterização de Moringa oleifera variedade óleo de semente "Periyakulam1". Journal of Food Composition and Analysis, $15,65-77$.

Leone, A., et al. (2016). Sementes e óleo de Moringa oleifera: Características e usos para a saúde humana. Revista Internacional de Ciências Moleculares, 17.

Logas, D., \& Kunkle, G. A. (1994). Double-blinded crossover study with marine oil supplementation containing high-dose icosapentaenoic acid for the treatment of canine pruritic skin disease. Veterinary Dermatology, 5(3), 99-104.

Macedo, L. C., et al. GF (2010). Prospecção tecnológica da Moringa oleifera Lam. In: II Encontro Nacional de Moringa, 1-3.

Mallya RPV NCPMp (2017). Moringa oleifera leaf extract: Beneficial effects on cadmium induced toxicities. A review. Journal of clinical and Diagnostic, 11(4), 01-04.

Makkar, H. P. S., \& Becker, K. (1996). Nutrional value and antinutritional components of whole and ethanol extracted Moringa oleifera leaves. Animal Feed Science Technology, 63, 211-228.

Martín, C., et al. (2013). Potential applications of Moringa oleifera. A critical review. Pastos y Forrajes, 36(2), $150-158$.

Matu, E. M., \& Van Staden, J. (2003). Antibacterial and anti-inflammatory activies of some plants used for medicinal purposes in Kenya. Journal Ethnopharmacol, 87(1), 35-41.

Mahmood, K. T., Mugal, T \& Haq, I. U (2010). Moringa oleifera: A natural gift-a review. Journal of Pharmaceutical Sciences and Research, 2(11), 775-781.

Miller, M. B., \& Bassler, B. L. (2001). Quorum sensing in bacteria. Annual Review of Microbiology, 55, 165-199.

Muller, M. R., et al. 2016). Evaluation of cyclosporine-sparing effects of polyunsaturated fatty acids in the treatment of canine atopic dermatitis. Veterinary Journal, 210, 77-81.

Muyibi, S. A., \& Evison, L. M (1995). Optimizing physical parameters affecting coagulation of turbid water with Moringa oleifera seeds. Watar Research, 29(12), 2689-2695.

Olivry, T., et al. (2010). Treatment of canine atopic dermatitis: 2010 clinical practice guidelines from the international task force on canine atopic dermatitis. Veterinary Dermatology, 21(3), 233-48. 
Research, Society and Development, v. 10, n. 8, e50010817495, 2021

(CC BY 4.0) | ISSN 2525-3409 | DOI: http://dx.doi.org/10.33448/rsd-v10i8.17495

Olson, M. F. (2013). Moringa olifera un árbol multiusos para la zonas tropicales secas. Mexicana biodiversidade, 82(4).

Proetti, S. (2018). As pesquisas qualitativa e quantitativa como métodos de investigação científica: Um estudo comparativo e objetivo. Revista Lumen -ISSN, 2(4), 2447-8717.

Qiu, S., et al. (2014). Natural alkaloids: basic aspects, biological roles, and future perspectives. Chinese Journal of Natural Medicines, 12(6), 401-406.

Rahman, M. M., et al. (2010). Control de bactérias coliformes detectadas en pacientes con diarrea asociada a extractos de Moringa oleifera. Nepal Medical College Journal, 12, 12-19.

Rego, E A. (2012). Avaliação da actividade anti-inflamatória de plantas dos açores. 119p. Dissertação [Mestrado em Ciências Biomédicas]. Departamento de Ciências Tecnológicas e Desenvolvimento, Universidade dos Açores, Ponta Delgada, Açores.

Rocha, M. F G., et al. Extratos de Moringa oleifera e Vernonia sp. sobre Candida albicans e Microsporum canis isolados de cães e gatos e análise da toxicidade em Artemia sp. Ciência Rural, 41(10), 1807-1812.

Shanker, K., et al. (2007). Determination of bioactive nitrile glycoside (s) in drumstick (Moringa oleifera) by reverse phase HPLC. Food Chemistry, 105(1), $376-382$.

Schuarz, D. (2000). Water purification using Moringa Oleifera. GATE Technical Information. 1.

Silva, A. K. S. (2010). Avaliação de atividades biológicas de flavonoides isolados da entrecasca de Sebastiania jacobinensis (MUILL. ARG.) MUILL. ARG. 62p. Dissertação [Mestrado em Bioquímica e Fisiologia]. Centro de Ciências Biológicas, Universidade Federal de Pernambuco, Recife, PE, Brasil.

Sulaiman, MRZZBASMIDMS (2008). Evaluatión of Moringa oleifera aqueous extract for antinociceptive and anti-inflammatory activities in animal models. Pharmaceutica Biology, 46(12), 838-845.

Scott, D. W., et al. (2001). Small animal dermatology. 6.ed. Philadelphia: W. B. Sauders Company, 667-779.

Sousa, C. A, \& Halliwell, R. E. W (2001). The ACVD task force on canine atopic dermatitis (XI): the relationship between arthropod hypersensitivity and atopic dermatitis in the dog. Veterinary Immunology and Immunopathology, 81(3-4), 233-237.

Valere, P. (2015). Medicinal plants in there west african. Ethopharmacologycal, 172, 297-311.

Vergara, J. M., et al. (2017). Bioactive components in Moringa oleifera leaves protect against chronic disease. Antioxidants, 6(4), 91.

White, P. D. (1998). Atopia. In: Bichard, S. J., Sherding, R. G. Manual saunders: clínica de pequenos animais, 343-351.

Zaku, S., et al. (2015). Moringa oleifera: An underutilized tree in Nigeria with amazing versatility: A review. African Journal Food Science, 9, 456-46.

Zaine, L., et al. (2014). Nutracêuticos imunomoduladores com potencial uso clínico para cães e gatos. Semina: Ciências Agrárias, 35(4), 2513-2530. 